\title{
Holographic Dark Energy with Curvature
}

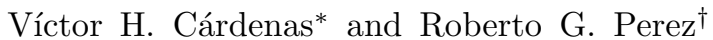 \\ Departamento de Física y Astronomía, Universidad de Valparaíso, Gran Bretaña 1111, Valparaíso, Chile
}

\begin{abstract}
In this paper we consider an holographic model of dark energy, where the length scale is the Hubble radius, in a non flat geometry. The model contains the possibility to alleviate the cosmic coincidence problem, and also incorporate a mechanism to obtain the transition from decelerated to an accelerated expansion regime. We derive an analytic form for the Hubble parameter in a non flat universe, and using it, we perform a Bayesian analysis of this model using SNIa, BAO and CMB data. We find from this analysis that the data favored a small value for $\Omega_{k}$, however high enough to still produce cosmological consequences.
\end{abstract}

PACS numbers: $98.80 . \mathrm{Cq}$

\section{INTRODUCTION}

One of the most important problems in cosmology is to explain the fact that the universe today is in a phase of accelerated expansion. In 1998, two teams studying distant Type Ia supernovae presented independent evidence that the expansion of the Universe is speeding up [1, 2]. The physical origin of cosmic acceleration remains a deep mystery. Since the expansion is speeding up, we are faced with two possibilities, either of which would have profound implications for our understanding of the cosmos and the laws of physics. The first is that $75 \%$ of the energy density of the Universe exists in a new form with large negative pressure, called dark energy. The other possibility is that General Relativity breaks down on cosmological scales and must be replaced with a more complete theory of gravity. Among the models proposed so far for dark energy, the Holographic Dark Energy (HDE) scenario emerge as one of the few that has a deep physical substratum.

The idea of Holography was first suggested by Bekenstein [3], by generalizing the results obtained from black hole physics, and postulating that the maximum entropy in a box of volume $L^{3}$ should growth as the area of the box. In this context, 'tHooft [4] and Susskind [5] stressed that this principle has to be considered as a recipe for a quantum gravity theory. The idea soon finds its way in cosmology [6]. The Holographic Principle can be described as follows: if we want to reconcile quantum mechanics with gravity, we have to assume that the observable degrees of freedom of the universe are projections coming from a two-dimensional surface, where the information is stored.

Considering that particle physics can be successfully described by an effective field theory with an ultraviolet (UV) cutoff, and black hole physics impose an infrared (IR) cutoff to the energy density involved, Cohen et al. [7] suggested that the dark energy should satisfy this prin-

\footnotetext{
${ }^{*}$ Electronic address: victor[at]dfa.uv.cl

${ }^{\dagger}$ Electronic address: genaro.perez[at]gmail.com
}

ciple, proposing a relationship between cutoff as

$$
L^{3} \rho_{\Lambda} \leq L M_{p}^{2},
$$

where $\rho_{\Lambda}$ is the dark energy density, $L$ the size of the system, and $M_{p}=(8 \pi G)^{-1 / 2}$ is the reduced Planck mass. In [8] the author notice that using $L$ as the size of the observed universe $\left(L_{\text {today }} \simeq 10 \mathrm{~Gy}\right)$ yields a value $\rho_{\Lambda} \simeq$ $10^{-10} \mathrm{eV}^{4}$, which would explain the observed value of the dark energy density. However, Hsu [9] demonstrated that although identifying $L$ with the Hubble radius $H^{-1}$ leads to a correct order of magnitude for the dark energy density, written $\rho_{\Lambda}=3 c^{2} H^{2} M_{p}^{2}$ saturating (11) with $c$ constant, this leads to a wrong equation of state. Based on this, Li [10] proposed instead to identify $L$ with the future event horizon.

Soon after, Pavon and Zimdahl [1] showed that is still possible to consider the Hubble radius as a length scale in the context of interacting dark energy models [12], which allows, as a bonus, to alleviate the cosmic coincidence problem: why the energy density of matter and dark energy are of the same order of magnitude just today. This time however, $\rho_{\Lambda}=3 c^{2} H^{2} M_{p}^{2}$ can actually describe the dark energy density assuming that $c$ is a time dependent function. The analysis performed in that paper was extended in [13] for non-flat cosmologies, focusing in the evolution of the ratio of the energy densities $r \equiv \rho_{m} / \rho_{\Lambda}$. An statistical analysis of this model in a flat universe was performed recently in [14].

In this paper we consider an interacting holographic dark energy model, using the Hubble radius as the length scale, in a universe with arbitrary curvature. The model not only contains the possibility to alleviate the coincidence problem, but also incorporate a mechanism to obtain the transition from decelerated to an accelerated expansion regime. We are primary interested in study the effects of the inclusion of a small curvature in the theoretical parameters in light of the observational data. We derive an analytic form for the Hubble parameter in a non flat universe, and using it, we perform a Bayesian analysis of this model using supernovae data, Baryon Acoustic Oscillation (BAO) distance, and the CMB shift parameter. We find from this analysis that the data favored a small value for $\Omega_{k}$, however high enough to produce important cosmological consequences [15]. 
In the next section we review the model clarifying the assumptions made in [13] and [14]. In section III we derive the flat universe results, some of them well known, and others new. The main contribution is explained in section IV where is derived the general solution for the non flat case. Next, we perform the bayesian analysis using observational data, and we end the paper with some comments.

\section{AN EFFECTIVE EQUATION OF STATE FOR DARK ENERGY}

The Einstein field equations for a homogeneous and isotropic universe are the Friedman equation

$$
H^{2}+\frac{k}{a^{2}}=\frac{1}{3 M_{p}^{2}} \rho,
$$

where $\rho$ is the energy density, $H=\dot{a} / a$ and $a(t)$ is the scale factor, $k=+1,0,-1$ for a closed, flat, and open geometries respectively, and

$$
\dot{H}=-\frac{1}{2 M_{p}^{2}}(\rho+P)+\frac{k}{a^{2}},
$$

where $P$ is the pressure, and where a dot means cosmic time derivative. The model emerges by assuming that the energy density $\rho$, which satisfy the usual energy conservation equation

$$
\dot{\rho}+3 H(\rho+P)=0,
$$

can be separated into two components; dark matter and dark energy, so $\rho=\rho_{m}+\rho_{\Lambda}$ and $P=p_{m}+p_{\Lambda}$. Assuming explicitly the equation of state for dark matter, $p_{m}=0$, and for dark energy $p_{\Lambda}=w_{\Lambda} \rho_{\Lambda}$, and further, considering that neither component conserves separately, we obtain

$$
\begin{array}{r}
\dot{\rho}_{\Lambda}+3 H \rho_{\Lambda}\left(1+w_{\Lambda}\right)=-Q, \\
\dot{\rho}_{m}+3 H \rho_{m}=+Q,
\end{array}
$$

where $Q$ is an arbitrary interaction term between dark matter and dark energy. From (5) we can take the $Q$ term to the left hand side and write

$$
\dot{\rho}_{\Lambda}+3 H\left(\rho_{\Lambda}+p_{\Lambda}+\frac{Q}{3 H}\right)=0,
$$

which looks like the energy conservation equation for dark energy but with an effective pressure given by

$$
p_{\Lambda}^{e f f}=p_{\Lambda}+\frac{Q}{3 H} .
$$

Then, we can write an effective equation of state for this dark energy as

$$
\omega_{\Lambda}^{e f f}=\frac{p_{\Lambda}^{e f f}}{\rho_{\Lambda}}=w_{\Lambda}+\frac{Q}{3 H \rho_{\Lambda}} .
$$

We can also rewrite this equation by using (5) and (6) obtaining

$$
\omega_{\Lambda}^{e f f}=-1-\frac{1}{3} \frac{d \ln \rho_{\Lambda}}{d \ln a},
$$

independent of the value of $w_{\Lambda}$.

\section{THE HOLOGRAPHIC MODEL FOR A FLAT UNIVERSE}

As a warm up and also to introduce the formalism, let us discuss the flat model first. This section is a critical review of previous works [11], [13] y [14].

\section{A. Constant c}

As we stated in the introduction, the corresponding vacuum energy density can be written from the holographic dark energy as

$$
\rho_{\Lambda}=3 c^{2} M_{p}^{2} H^{2},
$$

where $c$ is considered constant. Combining this with the Friedman equation enable us to write the energy density of dark matter

$$
\rho_{m}=3 M_{p}^{2} H^{2}\left(1-c^{2}\right) .
$$

To protect the positivity of the dark matter energy density, $c^{2}<1$ is required. Clearly, if $c$ is constant and the two components evolve independently (i.e. interaction $Q=0$ ), then $\rho_{m} \simeq H^{2} \simeq a^{-3}$ as the usual dark matter, but this implies also that $\rho_{\Lambda} \simeq a^{-3}$, an evolution that can not generate accelerated expansion. This is the result found by $\mathrm{Hsu}$ [9], [10].

However, as first stated in [1]], if we consider an interaction between dark matter and dark energy (see Eqs.(5) and (6) ), we can obtain an equation of state for dark energy different from that of dust. This is exactly what we have derived in the previous section in Eq.(10). Because $H=\dot{a} / a$ and using Eq.(4), we can find the dark energy density as a function of the scale factor

$$
\rho_{\Lambda}=\frac{c^{2}}{1-c^{2}} \rho_{m} \sim a^{-3\left(1-c^{2}\right)},
$$

In this case the deceleration parameter is

$$
q=\frac{1}{2}\left(1-3 c^{2}\right)
$$

To obtain an accelerated expansion universe, i.e. $q<$ 0 , and to protect the positivity of dark matter energy density, one obtains a range of values the constant $c$ can take: $1 / 3<c^{2}<1$. The effective equation of state of vacuum energy density is

$$
\begin{aligned}
w_{\Lambda}^{e f f} & =-1-\frac{1}{3} \frac{d \ln \rho_{\Lambda}}{d \ln a} \\
& =-c^{2} .
\end{aligned}
$$


Relation (13) indicates that the ratio $r \equiv \rho_{m} / \rho_{\Lambda}=$ $\left(1-c^{2}\right) / c^{2}$ is a constant. Although it is desirable to get a nearly constant $r$ parameter, solving the coincidence problem, getting a constant value, produces another problem: there is no transition from decelerated to an accelerated expansion regime.

\section{B. Variable c}

As we notice at the end of the previous section it is clear that when $c$ is constant, no transition from decelerated expansion to accelerated expansion can be realized, a transition observationally tested. A possible way to overcome this difficulty was proposed in [11]. The idea is to consider $c$ as a time dependent parameter, enabling also that $r=\left(1-c^{2}\right) / c^{2}$ to be. Since $r$ is expected to decrease with time, this implies that $c^{2}$ increases, keeping the relation $c^{2}(1+r)=1$.

In this case

$$
\rho_{\Lambda}=3 c^{2}(t) M_{P}^{2} H^{2} .
$$

and also, using the Friedman equation, we can write the relation

$$
\rho_{m}=3 M_{P}^{2}\left(1-c^{2}(t)\right) H^{2} .
$$

Notice that, although the redshift dependence of $\rho_{\Lambda}$ is not proportional to $H^{2}$, no further constraint on $c^{2}(z)$ is derived from the Friedman equation, because $\rho_{m}$ in (17) is defined to satisfy it automatically. Again, it is to protect the positivity of the energy density of cold dark matter that $c(t)^{2}<1$ is required. From the conservation equation of cold dark matter Eq. (6) and the Friedmann equation, one can write in a flat universe

$$
(1+z) \frac{d \ln H(z)}{d z}=\frac{3}{2}\left(1+w_{\Lambda} c^{2}(z)\right) .
$$

To solve Eq.(18), one has to assume a concrete form of the function $c(z)$. After a simple calculation, one can also write the deceleration parameter

$$
q=\frac{1}{2}\left(1-3 c^{2}(z)\right) .
$$

One finds that once $0<c^{2}(z)<1$ is time dependent, possible transition from deceleration expansion to accelerated expansion can be realized. The analogous expression for the effective equation of state in this case, can be derived using Eqs. (2) and (16), obtaining

$$
w_{\Lambda}^{e f f}=w_{\Lambda} c^{2}-\frac{1}{3} \frac{d \ln c^{2}}{d \ln a} .
$$

Combining this equation with (9) we obtain

$$
w_{\Lambda}=-\left(\frac{1+r}{r}\right)\left[\frac{Q}{3 H \rho_{\Lambda}}+\frac{\left(c^{2}\right)^{\cdot}}{3 H c^{2}}\right]
$$

a relationship first written in [1] , that shows how the equation of state parameter depends explicitly on the interaction and the $c^{2}(t)$ function.

If we write $Q=\Gamma \rho_{\Lambda}$, with $\Gamma$ being some particle production rate, we can write a solution for (6)

$$
\rho_{m}=\rho_{m}^{0}\left(\frac{a_{0}}{a}\right)^{3} \exp \left[\int \frac{\Gamma}{r} d t\right],
$$

where the subscript indicates today. An explicit model for a flat universe and interaction was proposed in [13], where it was suggested that

$$
\frac{\Gamma}{r H}=3 \beta\left(\frac{a}{a_{0}}\right)^{\alpha},
$$

in such a way that (22) can be solved exactly. In this case

$$
\frac{\rho_{m}}{\rho_{m}^{0}}=\left(\frac{a_{0}}{a}\right)^{3} \exp \left[\frac{3 \beta}{\alpha}\left(\left(\frac{a}{a_{0}}\right)^{\alpha}-1\right)\right] \text {. }
$$

If we assumed that $c^{2}$ is constant, then $\rho_{\Lambda}$ evolve in the same way as $\rho_{m}$, so we can explicitly write down the Hubble parameter from the Friedman equation. The deceleration parameter can also be computed being

$$
q=\frac{1}{2}-\frac{3}{2} \beta\left(\frac{a}{a_{0}}\right)^{\alpha} .
$$

An identical expression for $q$ was derived in [14], where instead a explicit form of $c^{2}(z)$ was given

$$
c^{2}(z)=\frac{\beta}{(1+z)^{\alpha}} .
$$

The equivalence between the models can be easily explained using the equation (21). The analysis performed in [14] consider a flat universe $k=0$ with interaction and is explicitly used that $w_{\Lambda}=-1$. This last assumption implies a relation between the interaction $Q$ and $c^{2}$. Actually, up to a constant, the relation is $c^{2}=Q /\left(3 r H \rho_{\Lambda}\right)$. The analysis performed both in [13] and 14] correspond to this model, considering interaction between the dark constituents in a flat universe, keeping the value $c^{2}(1+r)=1$.

\section{NON FLAT MODEL}

In this section we proceed to generalize the previous work to non flat universes. Although it is often said that the inflationary scenario predicts a flat universe [16], the situation has not been settled down yet, partly because the observations are not conclusive and because in theory $\Omega_{k}=0\left(\Omega_{k}=-k / a^{2} H^{2}\right.$ in this paper $)$ is not the only solution [17]. Moreover, in the context of parameter estimations, considering the curvature as a new free parameter, leads to an increment in the uncertainties in all the others cosmological parameters. In particular, in the 
context of models with dynamical dark energy, a number of works have discussed the degeneracies between $w(z)$ and $\Omega_{k}$ [18], [19], [20]. Therefore, the precision in observational cosmology requires the inclusion of the curvature, even if its contribution is small, in any theoretical study of dark energy. In this section $c^{2}$ is a function of time and $\Omega_{k} \neq 0$.

Let us start with Eq. (3). Replacing the definitions of both $\rho_{m}$ and $\rho_{\Lambda}$ we found

$$
\dot{H}=-\frac{3}{2} H^{2}\left(1+w_{\Lambda} c^{2}\right)-\frac{k}{2 a^{2}} .
$$

Using that $a=(1+z)^{-1}$ we can rewrite this equation using the redshift as the independent variable

$$
(1+z) \frac{d H^{2}}{d z}=3 H^{2}\left(1+w_{\Lambda} c^{2}\right)+k(1+z)^{2},
$$

which is the generalization of (18). The generalization of (21) follows in the same way as

$$
w_{\Lambda}=-\left(\frac{1+r}{r+\Omega_{k}}\right)\left[\frac{Q}{3 H \rho_{\Lambda}}+\frac{\Omega_{k}}{3}+\frac{\left(c^{2}\right)^{\cdot}}{3 H c^{2}}\right],
$$

that contains the obvious limit $\Omega_{k}=0$ result (21), and also coincides with the result given in [13] (see equation (26) in that paper). To show the equivalence we use the identities $\Omega_{m}+\Omega_{k}+\Omega_{\Lambda}=1$ and $r=\Omega_{m} / \Omega_{\Lambda}$. The analog of equation (20) in this case shows how the presence of curvature changes the dynamics. The effective equation of state parameter when $w_{\Lambda}=-1$ takes the form

$$
w_{\Lambda}^{e f f}=-c^{2}-\frac{\Omega_{k}}{3}-\frac{1}{3} \frac{d \ln c^{2}}{d \ln a} .
$$

The presence of curvature can ameliorate the negativity of $w_{\Lambda}^{\text {eff }}$ or, if it is positive, it can be even more negative, crossing the phantom line. However, the statistical analysis performed on this model, that we describe below, shows that because the curvature parameter turns out to be very small (see results in Table I), the actual value of $\Omega_{k}$ has a little impact on the dynamics of $w_{\Lambda}^{\text {eff }}$ (see Figure 2).

An explicit solution of (28) is possible when we fix the bare equation of state parameter $w_{\Lambda}=-1$

$$
\begin{array}{r}
H^{2}(z)=H_{0}^{2}(1+z)^{3} e^{\frac{3 c^{2}}{\alpha}} \times \\
{\left[e^{-\frac{3 \beta}{\alpha}}+\frac{\Omega_{k}}{\beta}\left(\frac{3 \beta}{\alpha}\right)^{-\frac{1}{\alpha}}\left\{\Gamma\left(\frac{1}{\alpha}, \frac{3 c^{2}}{\alpha}\right)-\Gamma\left(\frac{1}{\alpha}, \frac{3 \beta}{\alpha}\right)\right\}\right]}
\end{array}
$$

where $\Gamma(a, z)$ is the incomplete gamma function or plica function defined as

$$
\Gamma(a, z)=\int_{z}^{\infty} t^{a-1} e^{-t} d t
$$

Expression (31) for $\Omega_{k}=0$ reduces to the expression first written in [13] and contrasted with observations in [14].
TABLE I: The best fit values for the free parameters in the case of a non flat holographic dark universe. $\alpha$ and $\beta$ characterize the function $c^{2}(z)$ defined in (26) , and $\Omega_{k}$ is the curvature parameter. We specify the data set used in each .

\begin{tabular}{ccccc}
\hline \hline Data Set & $\chi_{\min }^{2}$ & $\alpha \pm 0.02$ & $\beta \pm 0.08$ & $\Omega_{k} \pm 0.009$ \\
\hline SN & 310.866 & 1.089 & 1.513 & -0.5873 \\
SN+BAO & 310.406 & 0.732 & 3.161 & 0.3876 \\
SN+BAO+CMB & 312.848 & 0.728 & 1.219 & -0.0449 \\
\hline \hline
\end{tabular}

Having the Hubble parameter in terms of the redshift, we can compute the luminosity distance and confront the model with observations of supernovae (SN) 22]. In this work, we use the Supernova Cosmology Project Union sample [21], having $307 \mathrm{SN}$ distributed over the range $0.015<z<1.551$. We fit the (theoretical) distance modulus $\mu(z)_{t h}$ defined by

$$
\mu(z)_{t h}=5 \log _{10}\left[\frac{H_{0} d_{L}(z)}{c}\right]+\mu_{0},
$$

to the observational ones $\mu(z)_{\text {obs }}$. Here $H_{0}=100 h \mathrm{~km}$ $\mathrm{s}^{-1} \mathrm{Mpc}^{-1}$ is the Hubble constant and the luminosity distance is defined by $d_{L}(z)=(1+z) r(z)$ where

$$
r(z)=\frac{c}{H_{0} \sqrt{\left|\Omega_{k}\right|}} \operatorname{Sinn} \sqrt{\left|\Omega_{k}\right|} \int_{0}^{z} \frac{d z^{\prime}}{H\left(z^{\prime}\right)},
$$

and $\mu_{0}=42.38-5 \log _{10} h . \operatorname{Sinn}(x)=\sin x, x, \sinh x$ for $\Omega_{k}<0, \Omega_{k}=0$, and $\Omega_{k}>0$ respectively. Considering $\alpha, \beta$ and $\Omega_{k}$ as free parameters, we get a $\chi_{\min }^{2}=310.866$ with the best fit values displayed in Table I.

The second major input for parameter determination comes from the baryon acoustic oscillations (BAO) detected by Eisenstein et al. [23]. In our work, we add the following term to the $\chi^{2}$ of the model:

$$
\chi_{B A O}^{2}=\left[\frac{\left(A-A_{B A O}\right)}{\sigma_{A}}\right]^{2},
$$

where $A$ is a distance parameter defined by

$$
\left.A=\frac{\sqrt{\Omega_{m} H_{0}^{2}}}{c z_{B A O}}\left[r^{2}\left(z_{B A O}\right) \frac{c z_{B A O}}{H\left(z_{B A O}\right)}\right)\right]^{1 / 3},
$$

and $A_{B A O}=0.469, \sigma_{A}=0.017$, and $z_{B A O}=0.35$. Considering both supernovae and BAO data, we obtain $\chi_{\min }^{2}=310.406$ with the best fit values displayed in the second row of Table I.

The CMB shift parameter $R$ is given by [24]

$$
R\left(z_{*}\right)=\sqrt{\Omega_{m} H_{0}^{2}} r\left(z_{*}\right)
$$

Here the redshift $z_{*}$ (the decoupling epoch of photons) is obtained by using the fitting function [25]

$$
z_{*}=1048\left[1+0.00124\left(\Omega_{b} h^{2}\right)^{-0.738}\right]\left[1+g_{1}\left(\Omega_{m} h^{2}\right)^{g_{2}}\right],
$$




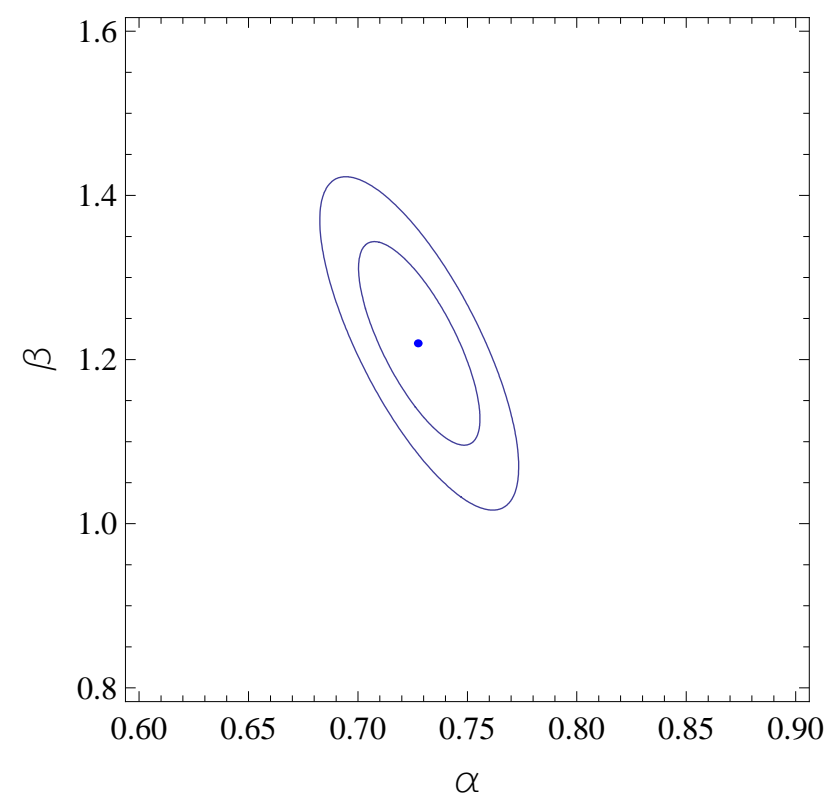

FIG. 1: These are the confidence contours in the plane $\alpha-\beta$ with $1 \sigma$ and $2 \sigma$ regions. The central dot shows the best fit parameter for the joint analysis using $\mathrm{SN}+\mathrm{BAO}+\mathrm{CMB}$ indicated in the third line of table I. This case correspond to a curvature parameter of $\Omega_{k}=-0.0449 \pm 0.009$.

where the functions $g_{1}$ and $g_{2}$ are given as

$$
\begin{aligned}
& g_{1}=0.0783\left(\Omega_{b} h^{2}\right)^{-0.238}\left(1+39.5\left(\Omega_{b} h^{2}\right)^{0.763}\right)^{-1}(39) \\
& g_{2}=0.560\left(1+21.1\left(\Omega_{b} h^{2}\right)^{1.81}\right)^{-1}
\end{aligned}
$$

The WMAP-7 year CMB data alone yields $R\left(z_{*}\right)=$ $1.726 \pm 0.018$ [26]. Defining the corresponding $\chi_{C M B}^{2}$ as

$$
\chi_{C M B}^{2}=\frac{\left(R\left(z_{*}\right)-1.726\right)^{2}}{0.018^{2}} .
$$

one can deduce constraints on $\alpha, \beta$ and $\Omega_{k}$. A joint analysis using $\mathrm{SN}+\mathrm{BAO}+\mathrm{CMB}$ leads to the best fit values showed in Table I. As is well known, we notice the effect of incorporating the BAO and CMB data to the supernova, leading to a decreasing value for $\Omega_{k}$. As in the $\Lambda \mathrm{CDM}$ model, when we use only the SNIa data, in particular the Union compilation, the fit suggest a huge curvature contribution (see figure 11 in [21] and the discussion). Only when we incorporate BAO and CMB constraint, the curvature parameter tends to smaller values. As is also well known, the strongest observational indication for a flat universe is the CMB data. A gaussian approximation to the confidence contour is also shown in Figure 1, where we show the plane $\alpha-\beta$ for $\Omega_{k}=-0.0449$. Using the best fit parameters we show in Figure 2 a plot of the resulting effective equation of state (30).

By choosing a cosmological constant equation of state $w_{\Lambda}=-1$, we get a model that, in agreement with observations, seems to cross the phantom line, leading to $w_{\Lambda}^{e f f}=-1$ for $z<1$.

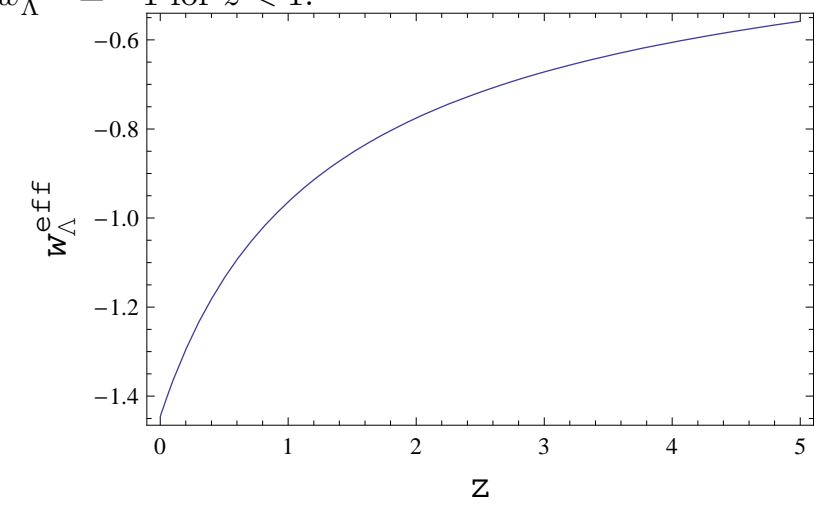

FIG. 2: Using the best fit parameters found in the analysis (see Table I), we plot the effective equation of state, expression (20), for our model. Notice that even if the bare equation of state parameter $w_{\Lambda}=-1$, the combined data is consistent with an effective equation of state that cross the cosmological constant line towards phantom like models.

\section{DISCUSSION}

We consider a non flat universe version of the holographic dark energy model studied first in [13] and [14], in which the interaction between dark matter and dark energy enable us to describe the transition from a decelerated to an accelerated expansion regime, together with alleviating the cosmic coincidence problem. Taking into account the spatial curvature, we were able to find an analytic expression for the Hubble parameter, which was used to perform a direct confrontation to observational data as SNIa, BAO and CMB shift parameter. This analysis shows that our model is compatible with current precision data. The best fit value for the curvature obtained, although small, it is well within the order where its presence can modify for example, the number of e-folding of inflation which changes dramatically the early stages of inflation [15].
[1] A.G. Riess, et al., Astron. J. 116 1009(1998) astro-ph/9805201.

[2] S. Perlmutter, et al., Astrophys. J. 517 565(1999) astro-ph/9812133.

[3] J. D. Bekenstein, Phys. Rev. D 7, 2333 (1973); Phys. Rev. D 23, 287 (1981).
[4] G. t Hooft, gr-qc/9310026, Published in Salamfestschrift: a collection of talks, eds. A. Ali, J. Ellis and S. Randjbar-Daemi (Worl Scientific).

[5] L. Susskind, J. Math. Phys. 36, 6377 (1995).

[6] W. Fischler and L. Susskind, hep-th/9806039; D. Bak and S.-J. Rey, Class. Quant. Grav. 17, L83 (2000); R. 
Tavakol and G. Ellis, Phys. Lett. B469, 37 (1999); R. Easther and D. Lowe, Phys. Rev. Lett. 82, 4967 (1999).

[7] A. Cohen, D. Kaplan and A. Nelson, Phys. Rev. Lett. 82 (1999) 4971.

[8] S. Thomas, Phys. Rev. Lett. 89 (2002) 081301.

[9] S.D.H. Hsu, Phys. Lett. B594 13(2004).

[10] M. Li, Phys. Lett. B603 1(2004).

[11] D. Pavon and W. Zimdahl, Phys. Lett. B628 206 (2005).

[12] B. Wang, C.Y. Lin, E.o Abdalla, Phys. Lett. B 637 357(2006); H. Kim, H.W. Lee, Y.S. Myung, Phys. Lett. B 632 605(2006); B. Hu, Y. Ling, Phys. Rev. D 73 123510(2006); H.M. Sadjadi, JCAP0702 026(2007); M.R. Setare, E.C. Vagenas, Int. J. Mod. Phys. D 18 147(2009); Q. Wu, Y. Gong, A. Wang, J.S. Alcaniz, arXiv:0705.1006; J.F. Zhang, X. Zhang, H.Y. Liu, Phys. Lett. B 659 26(2008); C. Feng, B. Wang, Y. Gong, R.K. Su, arXiv:0706.4033; S.F. Wu, P.M. Zhang, G.H. Yang, Class. Quan. Grav. 26 055020(2009).

[13] W. Zimdahl and D. Pavon, Class. Quant. Grav. 24, 5461 (2007); D. Pavon, J. Phys. A40, 6865 (2007).

[14] L. Xu, JCAP0909, 016 (2009).

[15] J-P.Uzan, U. Kirchner, G.F.R. Ellis, Mon. Not. Roy. Astron. Soc. 344, L65 (2003).

[16] V. Mukhanov, Physical Foundations of Cosmology, Cambridge (2005).
[17] A.Linde and A. Mezhlumian, Phys. Rev. D 52, 6789 (1995); A. Linde, D. Linde, A. Mezhlumian, Phys. Lett. B345, 203,(1995).

[18] E.V. Linder, Astropart. Phys. 24, 391 (2005).

[19] D. Polarski and A. Ranquet, Phys. Lett. B627, 1 (2005).

[20] C. Clarkson, M. Cortes and B. Bassett, JCAP 0708, 011 (2007).

[21] M. Kowalski et al., Astrophys. J. 686, 749(2008) arXiv:0804.4142.

[22] S. Nesseris and L. Perivolaropoulos, Phys. Rev. D 72, 123519 (2005) arXiv:astro-ph/0511040; L. Perivolaropoulos, Phys. Rev. D 71, 063503 (2005) arXiv:astro-ph/0412308; S. Nesseris and L. Perivolaropoulos, JCAP 0702, 025 (2007) arXiv:astro-ph/0612653.

[23] D. J. Eisenstein, et al, Astrophys. J. 633, 560 (2005) astro-ph/0501171.

[24] J. R. Bond, G. Efstathiou, and M. Tegmark, MNRAS 291 L33(1997).

[25] W. Hu, N. Sugiyama, Astrophys. J. 471 542(1996) astro-ph/9510117.

[26] E. Komatsu, et.al.,[WMAP Collaboration], arXiv:1001.4538 [astro-ph.CO]. 